Research Article

\title{
Synchronization of a Class of Chaotic Systems with Both Uncertainty and Disturbance by the UDE-Based Control Method
}

\author{
Zuoxun Wang (iD ${ }^{1,2}$ and Hongsheng Sha $\mathbb{D i D}^{1}$ \\ ${ }^{1}$ School of Electrical Engineering and Automation, Qilu University of Technology (Shandong Academy of Sciences), \\ Jinan 250353, China \\ ${ }^{2}$ High-Tech Industrial (Pilot) Base, Shandong Academy of Sciences, Jinan 250014, China \\ Correspondence should be addressed to Zuoxun Wang; wangzuoxun@126.com
}

Received 2 April 2020; Accepted 24 January 2021; Published 13 February 2021

Academic Editor: Luigi Rodino

Copyright (C) 2021 Zuoxun Wang and Hongsheng Sha. This is an open access article distributed under the Creative Commons Attribution License, which permits unrestricted use, distribution, and reproduction in any medium, provided the original work is properly cited.

\begin{abstract}
In this paper, chaotic synchronization with uncertainties and disturbances is studied. Firstly, a new control method based on UDE control is proposed, which is composed of two controllers: one is the stabilization controller in order to realize the stabilization of nominal system without uncertainties and disturbances and the other is the UDE controller in order to deal with the given controlled system with both uncertainties and disturbances. Then, two examples are studied by the above method. Finally, the correctness and effectiveness of the method are verified by numerical simulation.
\end{abstract}

\section{Introduction}

The chaotic synchronization phenomenon that caused great sensation in the academic world was first discovered by Pecora and Carrol in 1990 [1]. They realized the chaotic synchronization of the two same systems with different initial conditions in the electronics experiment. Decades later, they reviewed the literature on chaotic synchronization again [2]. Chaotic synchronization refers to the fact that the unstable motion controlled by the chaotic system tends to move in angular phase or amplitude with a similar rhythm through a certain coupling relationship and it has a wide range of applications in communications encryption, information science, chaotic generator design, and chemical reactions [3-8], etc. In recent years, scholars had proposed a variety of control methods to realize the synchronization between the same chaotic systems or the different chaotic systems with different initial conditions (refer to [9-20]). Among many control methods, the adaptive feedback control method [20] has a wide range of applications due to its simple design and easy implementation.

As far as we know, for chaotic synchronization, most of the existing control methods [9-13] only deal with the chaotic systems whose systems do not include model uncertainty and external disturbance and even if some methods can be used to cancel the uncertainty and disturbance, but the uncertainty and the disturbance are assumed bounded. As a matter of fact, uncertainty and the disturbance of such systems cannot be avoided and are often very large. Therefore, to investigate chaotic synchronization of the systems with both model uncertainty and disturbance is not only necessary, but also meaningful.

In order to solve this problem, the UDE controller [21-23], with its high bandwidth filter, can effectively remove the interference outside the system and it did have been widely used in the field of nonlinear control [22, 24]. However, this control method has some limitations in applications; for example, the controller's design is too complex to be realized in applications, which partly motivates our present work. Based on this, a new adaptive feedback control method based on UDE control is proposed by combining the adaptive feedback controller with the UDE controller to deal with synchronization problems of given chaotic system with both uncertainty and disturbance.

The remainder content of this paper is as follows: first of all, an adaptive feedback control method is given to solve the 
synchronization of given chaotic systems without uncertainty and disturbance. Then, the adaptive feedback controller is combined with the UDE controller to deal with synchronization problems of given chaotic system with both uncertainty and disturbance. At last, the simulation results verify the effectiveness and feasibility of the proposed control method.

The main structure of the following article is divided into three parts:

(1) Firstly, an adaptive feedback control method is given to solve the synchronization of given chaotic systems without uncertainty and disturbance

(2) Secondly, the adaptive feedback controller is combined with the UDE controller to deal with synchronization problems of given chaotic system with both uncertainty and disturbance

(3) Finally, the simulation results verify the effectiveness and feasibility of the proposed control method

\section{Preliminary}

2.1. Adaptive Feedback Control Method. Consider the following nonlinear system

$$
\dot{w}=g(w)
$$

where $w \in R^{n}$ is the state vector and $g(w)$ is a continuous function.

Let system (1) be the master system, then the corresponding controlled slave system is given as follows:

$$
\dot{v}=g(v)+N u
$$

where $v \in R^{n}$ is the state vector, $N \in R^{n \times r}, r \geq 1$, and $u$ is the designed controller.

Let $q=v-w$, the controlled error system can be written as follows:

$$
\dot{q}=g(v)-g(w)+N u
$$

Definition 1. Consider system (3). If $\lim _{t \rightarrow \infty}\|q(t)\|=0$, the master system (1) will synchronize the slave system (2) by the controller $u$.

Next, according to the existing result [20], let us briefly introduce the adaptive feedback controller.

$$
u_{s}=K q
$$

where $K=m(t) N^{T}$, and

$$
\dot{m}(t)=-o\|q(t)\|^{2},
$$

where $o>0$ is a constant.

Lemma 1. (see [20]). Consider the controlled error system (3), if $N=\left(N_{i j}\right)_{n \times r}$ and $N_{i j}=0$ or $N_{i j}=1, i=1,2, \ldots, n$ and $j=1,2, \ldots, r$, and $(g(v)-g(w), N)$ is controllable, then an adaptive controller $u_{s}$ is obtained as follows:
2.2. The UDE Controller. Since the UDE controller [22] is suitable for linear and nonlinear systems with uncertainties and perturbations, it is used by us to solve the problems of system uncertainty and perturbation. The following is a brief introduction of this control method.

Consider the following nonlinear system

$$
\dot{w}=h(w)+\Delta h(w)+N u+D(t),
$$

and $w \in R^{n}$ is the state vector, $N \in R^{n \times r}$ is a constant matrix, $\Delta h(w) \in R^{n}$ is the model uncertainty, and $D(t) \in R^{n}$ represents the external disturbance.

The stable linear reference model is described as follows:

$$
\dot{w}_{m}=Z_{m} w_{m}+N_{m} C,
$$

where $w_{m} \in R^{n}$ is the reference state vector, $Z_{m} \in R^{n \times n}$ is Hurwitz, $N_{m} \in R^{n \times r}$, and $C \in R^{r \times 1}$ is continuously consistent and bounded instructions for the system.

Lemma 2. (see [23]). Consider system (6), the designed filter $g_{A}(t)$ should satisfy

$$
\widetilde{u}_{d}=\widehat{u}_{d}-u_{d} \longrightarrow 0, \quad t \longrightarrow \infty,
$$

where $\widehat{u}_{d}=(\dot{w}-h(w)-N u) * s_{f}(t)$ and $u_{d}=\Delta h(w)+$ $D(t)$, and then, the UDE controller $u$ is designed as follows:

$$
\begin{aligned}
u= & N^{+}\left\{\ell^{-1}\left[\frac{1}{1-J_{f}(s)}\right] *\left(Z_{m} w+N_{m} C-K q\right)\right\} \\
& -N^{+}\left\{h(w)+\ell^{-1}\left[\frac{s J_{f}(s)}{1-J_{f}(s)}\right] * w(t)\right\},
\end{aligned}
$$

where $\ell^{-1}$ denotes the inverse Laplace transform operator, $N^{+}=\left(N^{T} N\right)^{-1} N^{T}, *$ is the convolution operator, and $J_{f}(s)=\ell\left[g_{f}(t)\right]$.

Remark 1. According to the existing result [22], the following two filters are often used in applications.

One is

$$
J_{f}(s)=\frac{1}{\tau s+1},
$$

in general, $\tau=0.001$

And the other is

$$
J_{f}(s)=\frac{a s+b-w_{0}^{2}}{s^{2}+a s+b},
$$

where $w_{0}=4 \pi, a=10 w_{0}$, and $b=100 w_{0}$.

\section{Main Results}

Consider the advantages of the adaptive control method and UDE control method, a new result of the combination of UDE controller and adaptive feedback controller is proposed.

Consider the following master chaotic system with both uncertainty and disturbance 


$$
\dot{w}=A(w)+\Delta A(w)+D(t)
$$

where $w \in R^{n}, \Delta A(w)$ denotes system model uncertainty, and $D(t)$ is external disturbance.

Then, the slave system is shown as follows:

$$
\dot{v}=A(v)+N u,
$$

where $v \in R^{n}$ is the state vector, $N=\left(b_{i j}\right)_{n \times r}$ and $b_{i j}=0$ or $b_{i j}=1, i=1,2, \ldots, n, j=1,2, \ldots, r$, and $u$ is the controller to be designed.

Let $q=v-w$, the error system is represented as follows:

$$
\dot{q}=A(v)-A(w)+\Delta A(w)+D(t)+N u,
$$

where $q \in R^{n}$ and $(A(v)-A(w), N)$ is controllable.

Then, we propose a conclusion.where $F(w, q)=$ $A(v)-A(w)+N u_{s}$.

Theorem 1. Consider the error system (14), if the designed filter $g_{A}(t)$ satisfies the following condition:

$$
\widetilde{u}_{d}=\widehat{u}_{d}-u_{d}, \quad t \longrightarrow \infty,
$$

where $\widehat{u}_{d}=\left(\dot{x}-A(w)-u_{d}\right) * g_{f}(t)$ and $u_{d}=\Delta A(w)+$ $D(t)$; and then, the UDE-based controller $u$ is shown as follows:

$$
u=u_{s}+u_{u d e},
$$

where $u_{s}=K q, K=m(t) N^{T}$, and

$$
\begin{aligned}
\dot{m}(t)= & -o\|e(t)\|^{2}, \\
u_{u d e}= & N^{+}\left\{\ell^{-1}\left[\frac{J_{f}}{1-J_{f}(s)}\right] * F(w, q)\right\} \\
& -N^{+}\left\{\ell^{-1}\left[\frac{s J_{f}(s)}{1-J_{A}(s)}\right] * q(t)\right\},
\end{aligned}
$$

Proof. Substituting $u$ in (16) to the error system (14), it results in $\dot{q}=A(v)-A(w)+N u_{s}+N u_{u d e}-u_{d}=$ $F(w, q)+N u_{u d e}-u_{d}$.

According to (15), it can be calculated as $B u_{u d e}=\widehat{u}_{d}$, and according to Lemma 1 , it can be concluded that the system $\dot{q}=F(w, q)$ is globally asymptotically stable. Thus, the conclusion is established.

\section{Illustrative Examples with Numerical Simulation}

In this section, the three-dimensional Lorenz chaotic system and 4D hyperchaotic system are taken as examples, respectively.

$$
\dot{w}=A(w)+\Delta A(w)+D(t)
$$

where $w=\left(w_{1}, w_{2}, w_{3}\right)^{T}, \Delta A(w)$ denotes system model uncertainty, and $D(t)$ is external disturbance, i.e.,

$$
A(w)=\left(\begin{array}{c}
A_{1}(w) \\
A_{2}(w) \\
A_{3}(w)
\end{array}\right)=\left(\begin{array}{c}
10\left(w_{2}-w_{1}\right), \\
28 w_{1}-w_{2}-w_{1} w_{3} \\
8 \\
-\frac{3}{3} w_{3}+w_{1} w_{2},
\end{array}\right)
$$

$$
\begin{aligned}
\Delta A(w) & =\left(\begin{array}{c}
0, \\
-0.1 w_{1}^{2}, \\
0,
\end{array}\right) \\
D(t) & =\left(\begin{array}{c}
0, \\
0.1 \sin (t) \\
0,
\end{array}\right)
\end{aligned}
$$

Example 1. Consider the following master chaotic system with both uncertainty and disturbance

Then, the slave system is shown as follows:

$$
\dot{v}=A(v)+N u,
$$

where $v=\left(v_{1}, v_{2}, v_{3}\right)^{T}$ and $(A(v)-A(v), N)$ is controllable, i.e.,

$$
\begin{aligned}
A(v) & =\left(\begin{array}{c}
A_{1}(v) \\
A_{2}(v) \\
A_{3}(v)
\end{array}\right)=\left(\begin{array}{c}
10\left(v_{2}-v_{1}\right), \\
28 v_{1}-v_{2}-v_{1} v_{3}, \\
8 \\
-\frac{1}{3} v_{3}+v_{1} v_{2},
\end{array}\right) \\
N & =\left(\begin{array}{l}
0 \\
1 \\
0
\end{array}\right) .
\end{aligned}
$$

Let $q=v-w$, the error system is shown as follows:

$$
\dot{q}=A(v)-A(w)+N u-\Delta A(w)-D(t),
$$

where $q=v-w \in R^{3}$ and $u=u_{s}+u_{u d e}$ is the controller to be designed. Our goal is to design a controller $u=u_{s}+u_{u d e}$ to stabilize system (25) with two control steps.

In the first step, the controller $u_{s}$ is to be designed as follows. By observing (20) and (23), if $q_{2}=0$, the following two-dimensional system

$$
\begin{aligned}
& \dot{q}_{1}=-28 q_{1}, \\
& \dot{q}_{3}=-\frac{8}{3} q_{3}+w_{2} q_{1}
\end{aligned}
$$

is globally asymptotically stable.

According to Lemma 2, the controller $u_{s}$ can be designed as follows:

$$
u_{s}=m(t) N^{T} q=m(t)(010)^{T} q
$$


and the feedback gain $m(t)$ is updated by the update law (17). Next, the numerical simulation is carried out with the initial conditions (for the convenience of the reader, we replace $w($.$) with x(),. v($.$) with y($.$) , and m($.$) with k($.$) , and$ the specific initial values are shown as follows: $x_{1}(0)=$ $0.1, x_{2}(0)=0.2, x_{3}(0)=0.3, y_{1}(0)=1, y_{2}(0)=2, y_{3}(0)=$ $3, k(0)=-1)$; then, the numerical simulation results are shown as follows.

Figure 1 shows that the error system is asymptotically stable, and Figure 2 shows that the feedback gain $k(t)$ converges to a negative constant.

The second step is to design the UDE controller $u_{\text {ude }}$. Let $u_{d}=\Delta A(w)+D(t), F(x)=A(v)-A(w)+N u_{s}$, and system (25) is rewritten as follows:

$$
\dot{w}=F(w)+N u_{u d e}-u_{d} .
$$

According to Lemma 2, the controller $u_{u d e}$ is designed as

$$
u_{\text {ude }}=N^{+}(\dot{w}-A(w)) * g_{f}(t) .
$$

Thus, the controller $u=u_{s}+u_{u d e}$ is completely designed. In the following, the simulation results is carried out as follows.

Figure 3 shows that the error system is also asymptotically stable, Figure 4 shows that the states $x_{1}, x_{2}, x_{3}$ synchronize the states $y_{1}, y_{2}, y_{3}$, respectively, Figure 5 shows that the feedback gain $k(t)$ converges to a negative constant, and Figure 6 shows that $\widehat{u}_{d}$ tends to $u_{d}$ as $t \longrightarrow \infty$.

Example 2. Consider the controlled 4D hyperchaotic system with both uncertainty and disturbance as follows:

$$
\dot{w}=A(w)+\Delta A(w)+D(t),
$$

where $w=\left(w_{1}, w_{2}, w_{3}, w_{4}\right)^{T}$.

$$
\begin{aligned}
A(w) & =\left(\begin{array}{l}
A_{1}(w) \\
A_{2}(w) \\
A_{3}(w) \\
A_{4}(w)
\end{array}\right)=\left(\begin{array}{c}
15\left(w_{2}-w_{1}\right), \\
10 w_{2}-w_{1} w_{3}+w_{4}, \\
w_{1} w_{2}-5 w_{3}, \\
w_{3}-w_{4},
\end{array}\right) \\
\Delta A(w) & =\left(\begin{array}{c}
0, \\
-0.1 w_{1}^{2} \\
0, \\
0, \\
0, \\
0.1 \sin (t), \\
0, \\
0,
\end{array}\right)
\end{aligned}
$$

Let system (30) be the master system, then the slave system is given as follows:

$$
\dot{v}=f(v)+N u,
$$

where $v=\left(v_{1}, v_{2}, v_{3}, v_{4}\right)^{T}$.

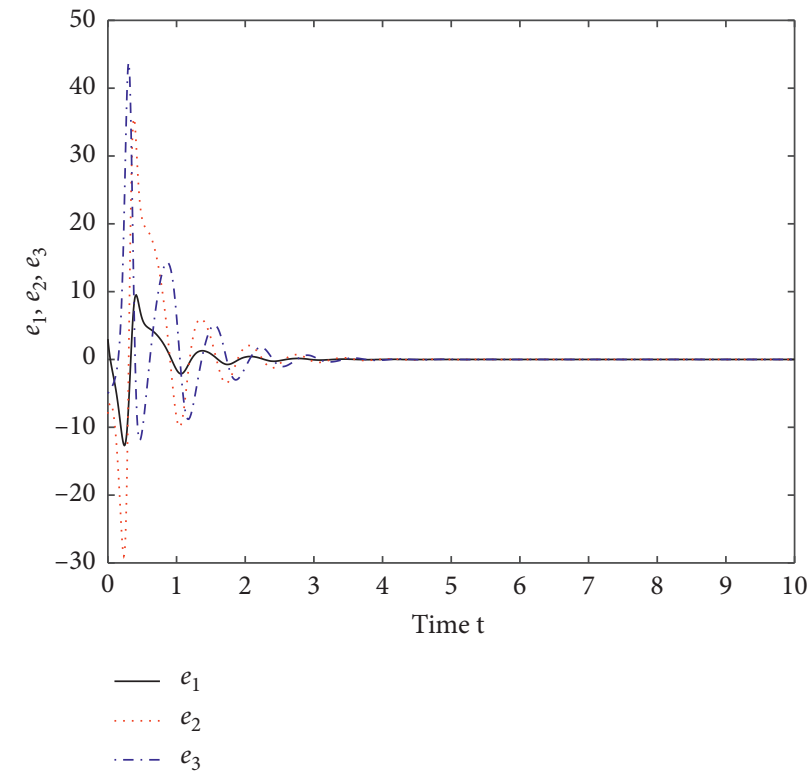

FIgURE 1: The error system is asymptotically stable.

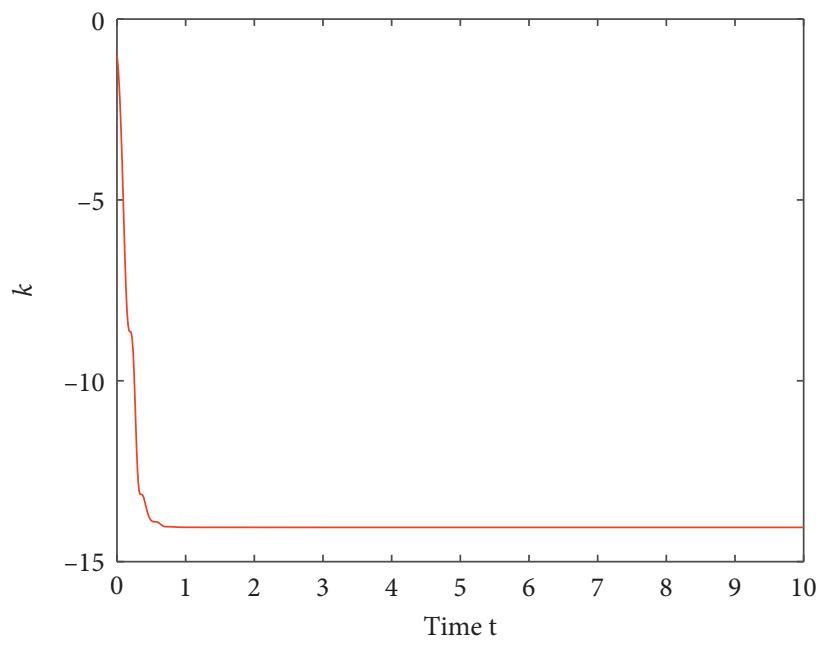

Figure 2: The feedback gain $k(t)$ converges to a negative constant.

$$
\begin{aligned}
A(v) & =\left(\begin{array}{l}
A_{1}(v) \\
A_{2}(v) \\
A_{3}(v) \\
A_{4}(v)
\end{array}\right)=\left(\begin{array}{c}
15\left(v_{2}-v_{1}\right) \\
10 v_{2}-v_{1} v_{3}+v_{4} \\
v_{1} v_{2}-5 v_{3} \\
v_{3}-v_{4}
\end{array}\right), \\
N & =\left(\begin{array}{ll}
0 & 0 \\
1 & 0 \\
0 & 1 \\
0 & 0
\end{array}\right) .
\end{aligned}
$$

Let $q=v-w$, the error system is represented as follows:

$$
\dot{q}=f(v)-f(w)+N u-\Delta A(w)-D(t),
$$

where $u=u_{s}+u_{u d e}$ is the controller to be designed. 

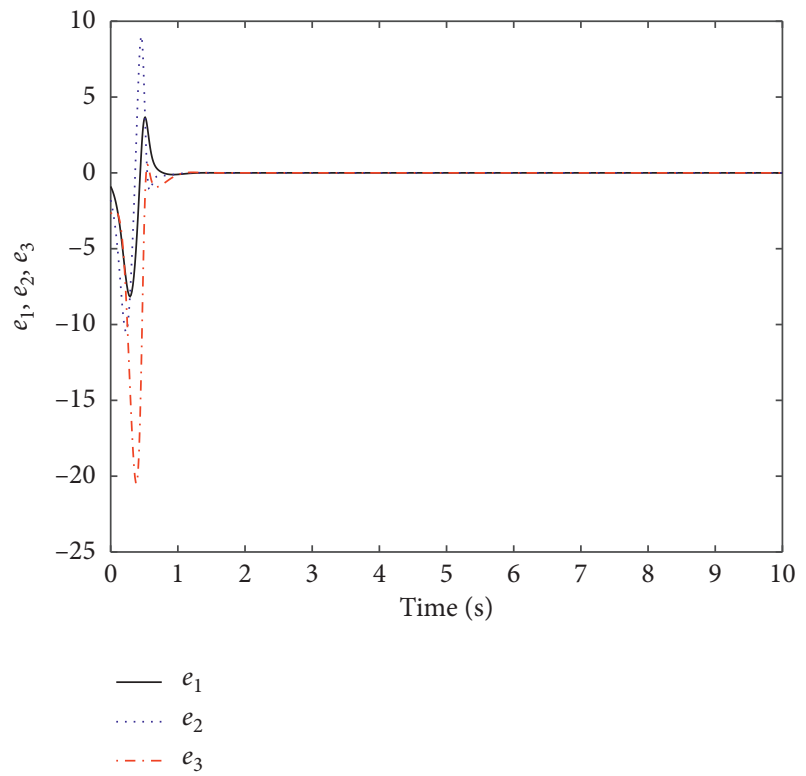

Figure 3: The error system is also asymptotically stable.
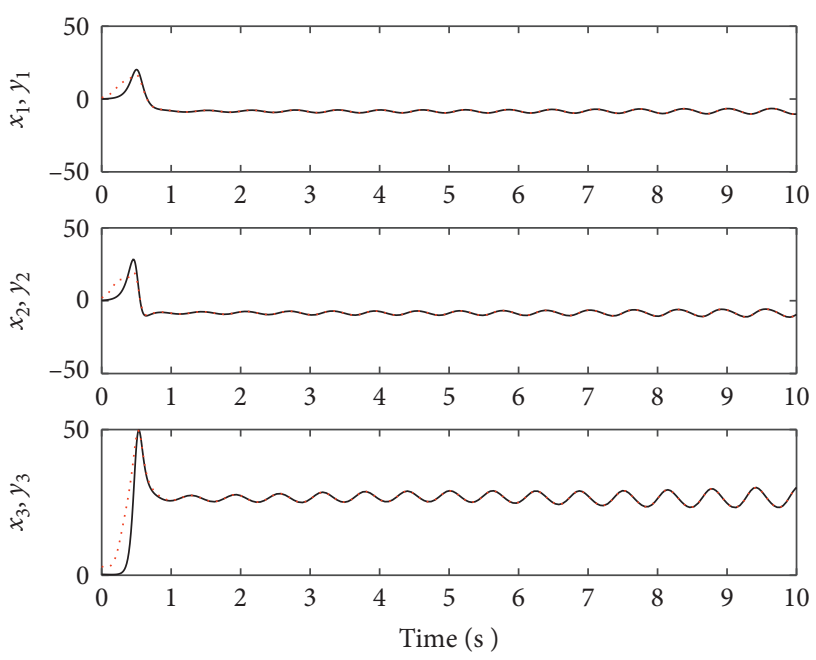

Figure 4: The states $x_{1}, x_{2}, x_{3}$ synchronize the states $y_{1}, y_{2}, y_{3}$, respectively.

Our goal is to design a controller $u=u_{s}+u_{u d e}$ to stabilize system (34) with two control steps.

In the first step, the controller $u_{s}$ is to be designed as follows.

By observing the error system (34), i.e.,

$$
\begin{aligned}
& \dot{q}_{1}=15\left(q_{2}-q_{1}\right), \\
& \dot{q}_{2}=10 q_{2}-q_{1} q_{3}+q_{4}-w_{3} q_{1}-w_{1} q_{3}, \\
& \dot{q}_{3}=q_{1} q_{2}-5 q_{3}+w_{1} q_{2}+w_{2} q_{1}, \\
& \dot{q}_{4}=q_{3}-q_{4} .
\end{aligned}
$$

By observing the conclusion results of (35), we note that, if $q_{2}=0, q_{3}=0$, then the following subsystem

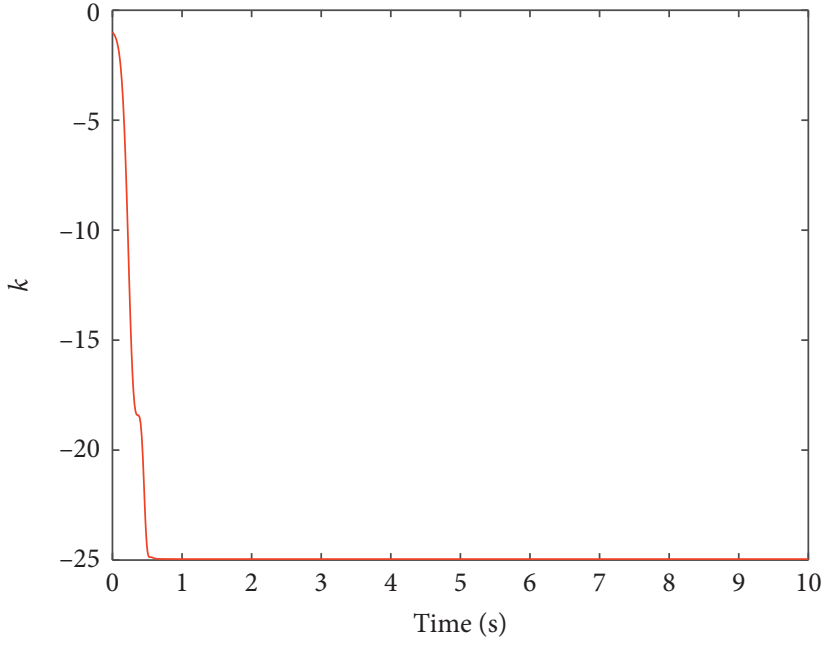

FIGURE 5: The feedback gain $k(t)$ converges to a negative constant.

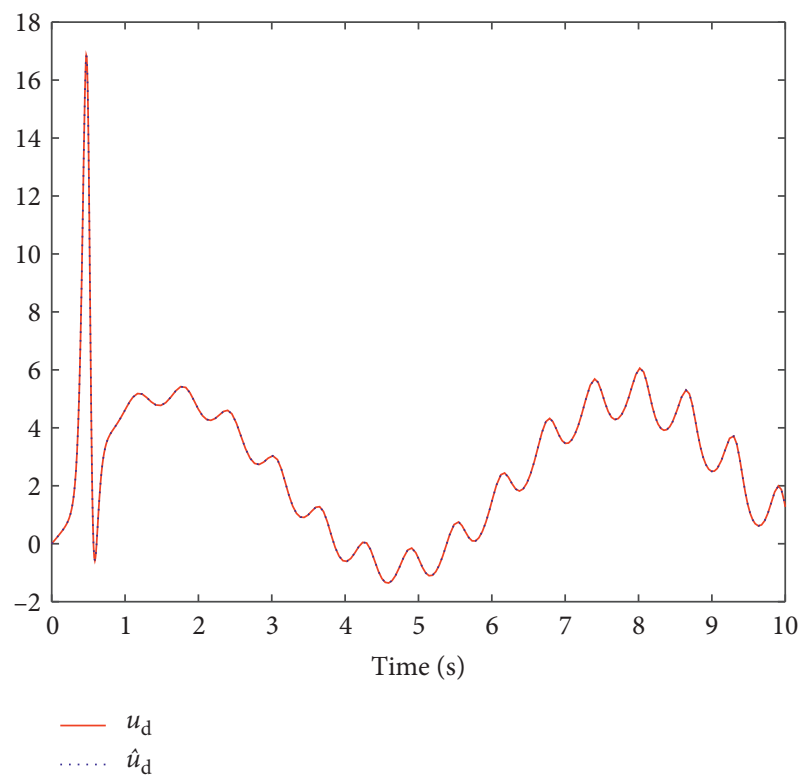

Figure 6: $\widehat{u}_{d}$ tends to $u_{d}$ as $t \longrightarrow \infty$.

$$
\begin{aligned}
& \dot{q}_{1}=-15 q_{1}, \\
& \dot{q}_{4}=-q_{4}
\end{aligned}
$$

is globally asymptotically stable.

Therefore, according to Lemma 1 , the controller $u_{s}$ can be designed as follows:

$$
u_{s}=m(t) N^{T} q=m(t)\left(\begin{array}{llll}
0 & 1 & 0 & 0 \\
0 & 0 & 1 & 0
\end{array}\right) q,
$$

and the feedback gain $m(t)$ is updated by the update law (17).

Then, the simulation result is carried out with the initial condition (for the convenience of the reader, we replace $w($. with $x(),. v($.$) with y($.$) , and m($.$) with k($.$) :$ $x_{1}(0)=1, x_{2}(0)=-2, x_{3}(0)=3, x_{4}(0)=-4, y_{1}(0)=5$, $\left.y_{2}(0)=-6, y_{3}(0)=7, y_{4}(0)=-8, k_{0}=-1\right)$. 


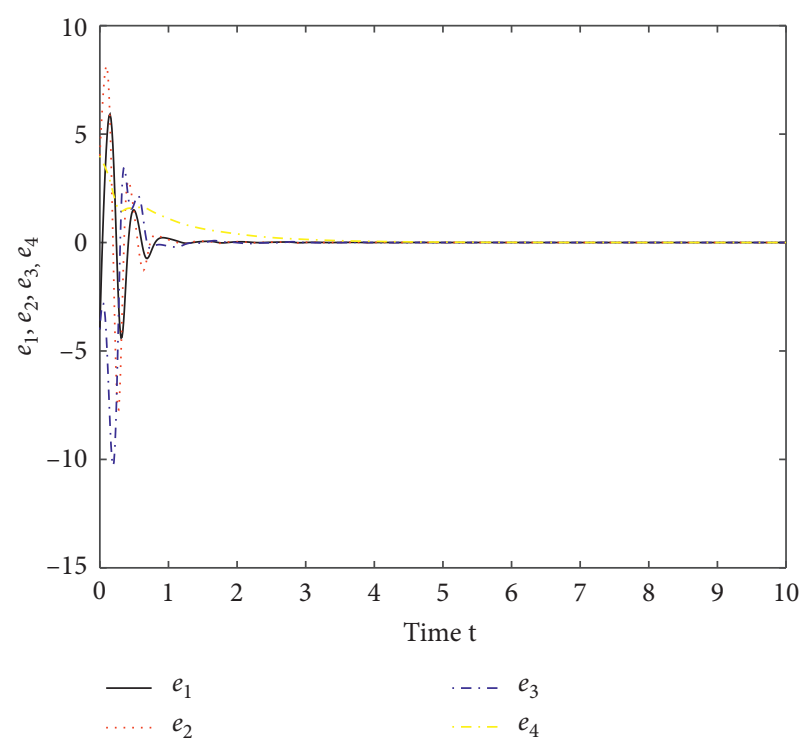

FIgURE 7: The error system is asymptotically stable.

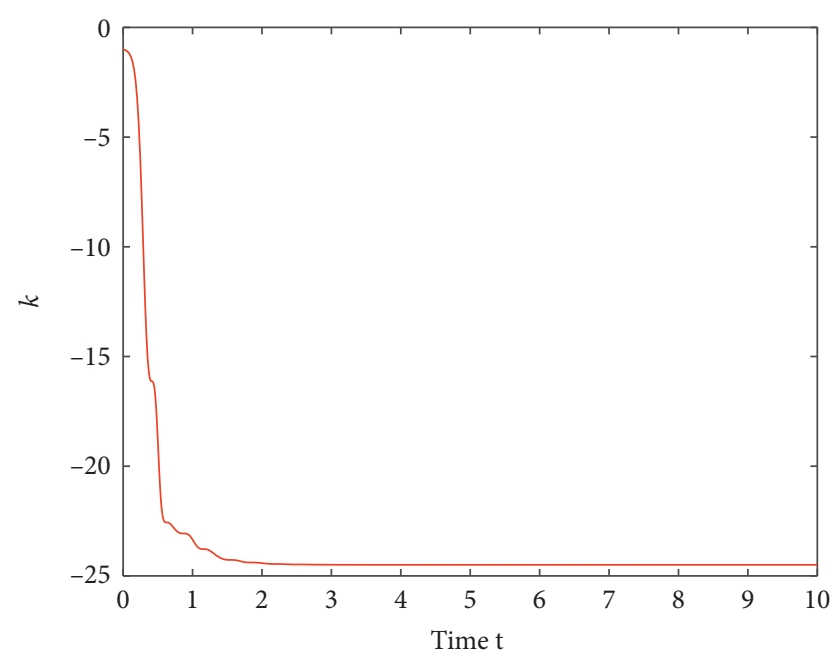

Figure 8: The feedback gain $k(t)$ converges to a negative constant.

Figure 7 shows that the error system is asymptotically stable, and Figure 8 shows that the feedback gain $k(t)$ converges to a negative constant.

In the second step, the controller $u_{u d e}$ is designed as follows.

$u_{d}=\Delta A(w)+D(t), F(w)=A(v)-A(w)+N u_{s}, \quad$ system (30) is rewritten as follows:

$$
\dot{w}=F(w)+N u_{u d e}-u_{d} .
$$

According to Lemma 2, the controller $u_{\text {ude }}$ can be designed as follows:

$$
\begin{aligned}
u_{\text {ude }}= & N^{+}\left\{\ell^{-1}\left[\frac{J_{A}(s)}{1-J_{A}(s)}\right] * F(w, q)\right\} \\
& -N^{+}\left\{\ell^{-1}\left[\frac{s J_{A}(s)}{1-J_{A}(s)}\right] * q(t)\right\},
\end{aligned}
$$

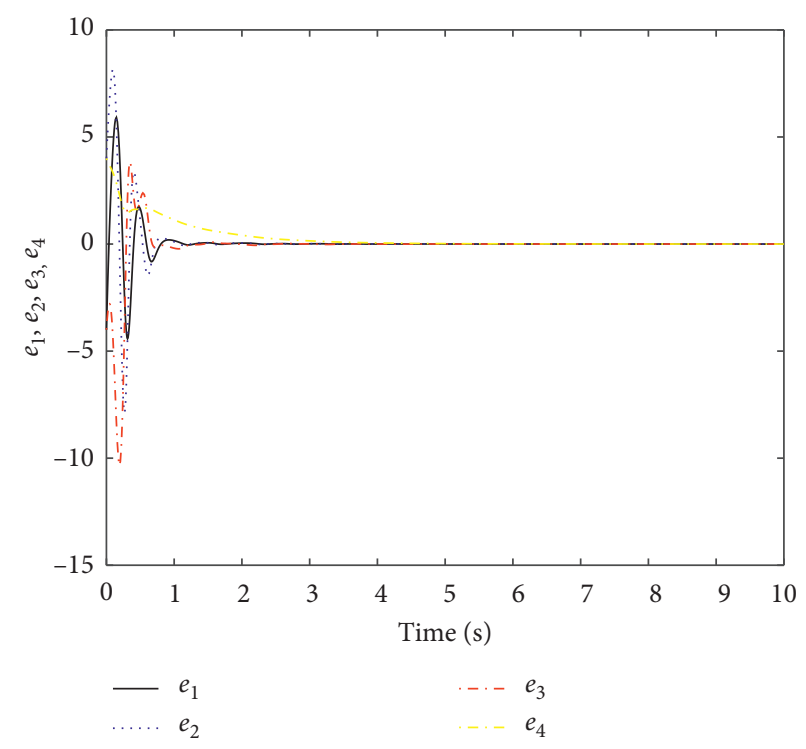

FIgURE 9: The error is also asymptotically stable.

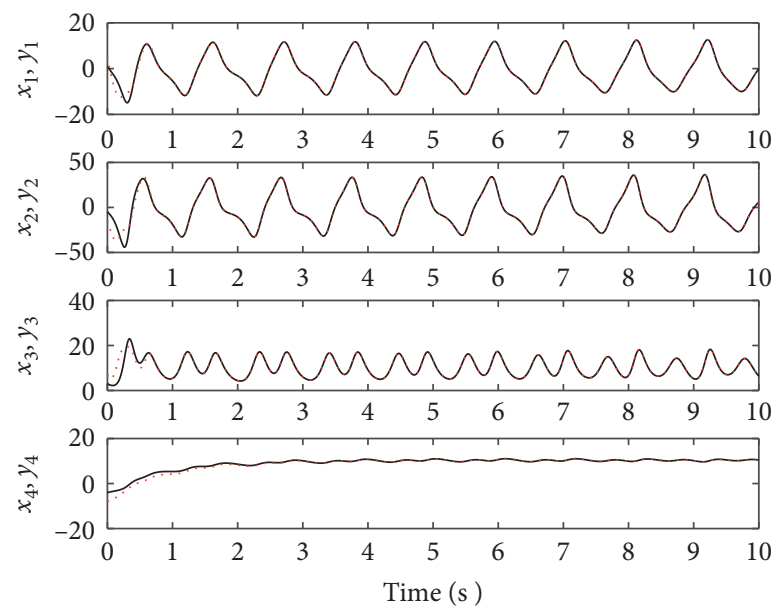

FIgURE 10: The states $x_{1}, x_{2}, x_{3}, x_{4}$ synchronize the states $y_{1}, y_{2}, y_{3}, y_{4}$, respectively.

where $\ell^{-1}$ denotes the inverse Laplace transform operator, $N^{+}=\left(N^{T} N\right)^{-1} N^{T}, *$ is the convolution operator, and $J_{A}(s)=\ell\left[g_{A}(t)\right]$. Thus, the controller $u=u_{s}+u_{u d e}$ is completely designed. Next, the simulation is carried out with the initial condition (for the convenience of the reader, we replace $w($.$) with x(),. v($.$) with y($.$) , and m($.$) with k($.$) :$ $x_{1}(0)=1, x_{2}(0)=-2, x_{3}(0)=$ $3, x_{4}(0)=-4, y_{1}(0)=5, y_{2}(0)=-6, y_{3}(0)=$ $\left.7, y_{4}(0)=-8, k_{0}=-1\right)$, and the numerical simulation results are shown in Figure 9-12.

Figure 9 shows that the error system is also asymptotically stable, Figure 10 shows that the states $x_{1}, x_{2}, x_{3}$ synchronize the states $y_{1}, y_{2}, y_{3}$, respectively, Figure 11 shows that the feedback gain $k(t)$ converges to a negative constant, and Figure 12 shows that $\widehat{u}_{d}$ tends to $u_{d}$ as $t \longrightarrow \infty$. 


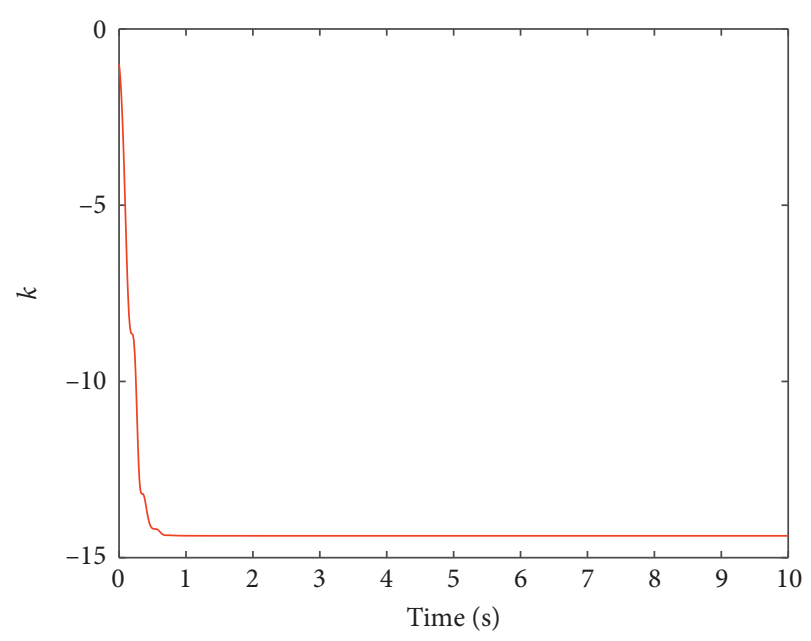

Figure 11: The feedback gain $k(t)$ converges to a negative constant.

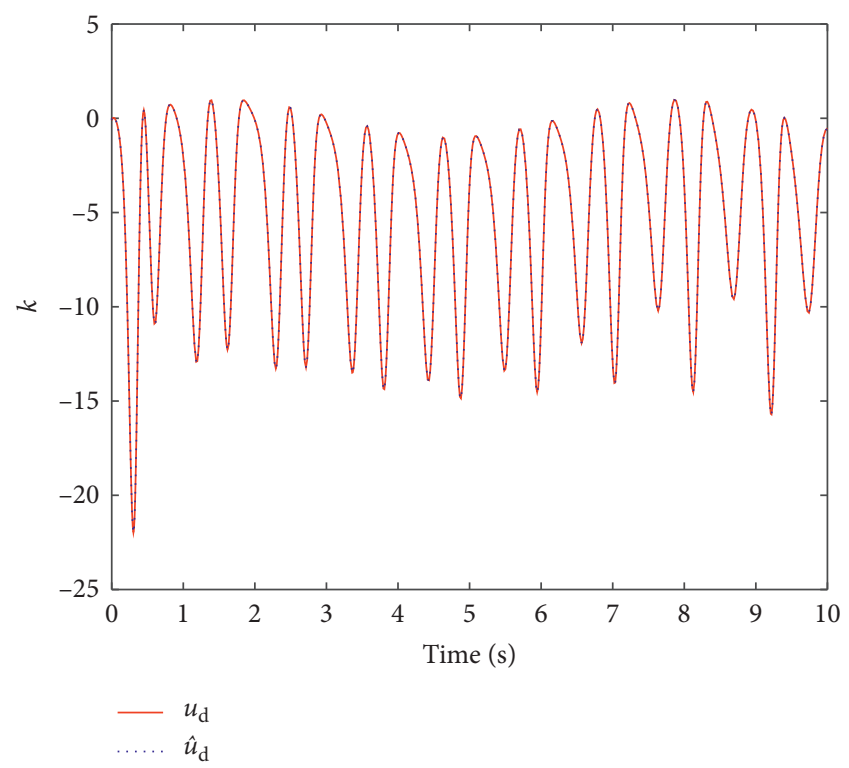

Figure 12: $\widehat{u}_{d}$ tends to $u_{d}$ as $t \longrightarrow \infty$.

\section{Conclusion}

In this paper, synchronization of chaotic systems with both uncertainty and disturbance has been investigated. Firstly, a new UDE-based control method has been presented, which is composed of two controllers: one is the stabilization controller and the other is the UDE controller. Secondly, two examples have been studied by the above methods. Finally, numerical simulations have verified the effectiveness and correctness of the theoretical results.

\section{Data Availability}

No data were used in this paper.

\section{Conflicts of Interest}

The authors declare that they have no financial and personal relationships with other people or organizations that can inappropriately influence our work, and there is no professional or other personal interest of any nature or kind in any product, service, and/or company that could be construed as influencing the position presented in, or the review.

\section{Acknowledgments}

This work was supported by Science, Education, and Industry Integration Innovation Pilot Project of Qilu University of Technology (Shandong Academy of Sciences) (2020KJC-ZD04).

\section{References}

[1] L. M. Pecora and T. L. Carroll, "Synchronization in chaotic systems," Physical Review Letters, vol. 64, no. 8, pp. 821-824, 1990.

[2] L. M. Pecora and T. L. Carroll, "Synchronization of chaotic systems," Chaos, vol. 25, no. 9, pp. 097-611, 2015.

[3] S. Bowong, "Stability analysis for the synchronization of chaotic systems with different order: application to secure communications," Physics Letter A, vol. 326, no. 2, pp. 102-113, 2004.

[4] R. R. Ojalvo, "Spatiotemporal communication with synchronized optical chaos," Physical Review Letter, vol. 86, no. 28, p. 5204, 2001.

[5] K. Murali and M. Lakshmanan, "Drive-response scenario of chaos synchronization in identical nonlinear systems," Physical Review E, vol. 49, no. 6, p. 4882, 1994.

[6] K. Murali and M. Lakshmanan, "Impulsive stabilization for control and synchronization of chaotic systems: theory and application to secure communication," Physical Review E, vol. 44, no. 10, pp. 976-988, 1997.

[7] Z.-L. Wang and X.-R. Shi, "Anti-synchronization of Liu system and Lorenz system with known or unknown parameters," Nonlinear Dynamics, vol. 57, no. 3, pp. 425-430, 2009.

[8] R. Guo, "Projective synchronization of a class of chaotic systems by dynamic feedback control method," Nonlinear Dynamics, vol. 90, no. 1, pp. 53-64, 2017.

[9] A. Yang, L. Li, Z. Wang, and R. Guo, "Tracking control of a class of chaotic systems," Symmetry, vol. 11, no. 4, p. 568, 2019.

[10] Z. Wang and R. Guo, "Hybrid synchronization problem of a class of chaotic systems by an universal control method," Symmetry, vol. 10, no. 11, p. 552, 2018.

[11] D. B. Huang, "Simple adaptive-feedback controller for identical chaos synchronization," Physical Review E, vol. 71, no. 3, pp. 037-203, 2005.

[12] D. B. Huang, "Adaptive-feedback control algorithm," Physical Review E, vol. 73, no. 6, pp. 066-204, 2006.

[13] D. B. Huang, "Synchronization in adaptive weighted networks," Physical Review E, vol. 74, no. 4, pp. 046-208, 2006.

[14] H. G. Zhang, Y. H. Xie, Z. L. Wang, and C. D. Zheng, "Adaptive synchronization between two different chaotic neural networks with time delay," IEEE Transaction on Network, vol. 18, no. 6, pp. 1841-1845, 2007.

[15] T. Yang and L. O. Chua, "Impulsive stabilization for control and synchronization of chaotic systems: theory and application to secure communication," IEEE Transations on Circuits Systems, vol. 44, no. 10, pp. 976-988, 1997.

[16] M. Chen, Q. Wu, and C. Jiang, "Disturbance-observer-based robust synchronization control of uncertain chaotic systems," Nonlinear Dynamics, vol. 70, no. 4, pp. 2421-2432, 2012. 
[17] T. Hou, Y. Liu, and F. Deng, "Finite horizon H2/Hoo control for SDEs with infinite Markovian jumps," Nonlinear Analysis: Hybrid Systems, vol. 34, pp. 108-120, 2019.

[18] H. Chen, J. Chang, J. Yan, and T. Liao, "EP-based PID control design for chaotic synchronization with application in secure communication," Expert Systems with Applications, vol. 34, no. 2, pp. 1169-1177, 2008.

[19] R. Xu and F. Zhang, " $\epsilon$-Nash mean-field games for general linear-quadratic systems with applications," Automatica, vol. 114, Article ID 108835, 2020.

[20] L. Liu, B. Li, and R. Guo, "Consensus control for networked manipulators with switched parameters and topologies," IEEE Access, vol. 9, p. 9209, 2021.

[21] B. Ren, Q.-C. Zhong, and J. Dai, "Asymptotic reference tracking and disturbance rejection of UDE-based robust control," IEEE Transactions on Industrial Electronics, vol. 64, no. 4, pp. 3166-3176, 2017.

[22] B. B. Ren, Q. C. Zhong, and J. H. Jinhao, "Robust control for a class of nonaffine nonlinear systems based on the uncertainty and disturbance estimator," IEEE Transactions on Industrial Electronics, vol. 62, no. 9, pp. 0278-0046, 2015.

[23] Q.-C. Zhong and D. Rees, "Control of uncertain LTI systems based on an uncertainty and disturbance estimator," Journal of Dynamic Systems, Measurement, and Control, vol. 126, no. 4, pp. 905-910, 2004.

[24] A. Kuperman and Q.-C. Zhong, "UDE-based linear robust control for a class of nonlinear systems with application to wing rock motion stabilization," Nonlinear Dynamics, vol. 81, no. 1-2, pp. 789-799, 2015. 